\title{
Troponin T Measurement
}

National Cancer Institute

\section{Source}

National Cancer Institute. Troponin T Measurement. NCI Thesaurus. Code C74750.

The determination of the amount of troponin T present in a blood sample. 